\title{
FABRICATION AND TESTING OF A LOW-COST FOOT PRESSURE SENSING SYSTEM
}

\author{
Jasim Ahmed Ali AL-Baghdadi ${ }^{\mathrm{a}, \mathrm{b}}$, Albert K. Chong ${ }^{\mathrm{b} *}$, Peter D. Milburn ${ }^{\mathrm{c}}$

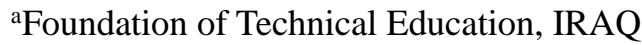 \\ bUniversity of Southern Queensland, Australia \\ ${ }^{\mathrm{C}}$ Griffith University, Australia \\ *Corresponding Author: chonga@usq.edu.au
}

\begin{abstract}
The main purpose of the research was to develop a lowcost plantar sensor system which can be used to capture human sole (plantar) pressure during the loaded phase of gait accurately. The known factors for causing the development of plantar pain, strain and ulcer were unusual high peak plantar pressure, high plantar pressure gradient and high plantar shear value during the support phase of gait. Precise pressure data is crucial in the monitoring and treatment of these illnesses. In this investigation, off-the-shelf pressure thin-sheet sensor pads were assembled, calibrated and tested for the acquisition of pressure data. Test results show that the low-cost system is suitable for high-quality pressure data acquisition. Therefore, low-cost thin-sheet pressure pads can be used to assemble large precise pressure sensing system for many applications such as in health and industry.
\end{abstract}

Keywords: pressure sensor, calibration, human gait.

\section{Introduction}

It is essential to determine and analyze human sole (plantar) pressure (pp) during the loaded phase of walking (gait) on hard substrate as this can provide valuable information on the characteristic of the pressure and forces that act on specific parts of the foot. These data can provide the insight into possible risk factors for chronic foot pain, foot ulcer and foot injury ${ }^{(1,2)}$. Plantar load distribution investigations emphasize the determination and analysis of pressures or forces acting on the plantar tissue during static or dynamic conditions ${ }^{(3,4,5)}$. Barefoot load distribution characteristic differ from person to person due to a range of factors such as the variations in the walking speed ${ }^{(5,6,7)}$, body weight $^{(5,8,9)}$, individual age ${ }^{(5,8)}$, foot geometry ${ }^{(10)}$ and stride length ${ }^{(5,8)}$. However, pacing velocity and the structural variations in the foot arch can have significant influences in changing the foot load distribution among people ${ }^{(5,6,7,11)}$.

Plantar loading has been studied in static ${ }^{(4)}$ and dynamic situations $^{(3,4,5,12)}$. To determine the load or pressure distribution exerted on the plantar surface, a number of investigations have divided the plantar surface into eight anatomical regions as shown in Figure $1^{(13,14)}$. It has been reported that during normal stance, each foot carries about half of the body weight ${ }^{(15,16)}$ at the heel, forefoot and big toe whereas lowest plantar load is located under the midfoot ${ }^{(1)}$. However, a PP distribution map alters noticeably during foot movement ${ }^{(6,7)}$. Previous literature has shown that an increase in the speed of foot movement leads to an increase in peak PP in the foot regions such as heel, medial and central metatarsals and hallux ${ }^{(1,4,5,17)}$. Hennig ${ }^{(17)}$ stated that the peak PP transfers sequentially from the heel to the metatarsal heads between the early and the late stance. For instance, Hayafune ${ }^{(18)}$ reported that the during late stance phase, the load acting on the first and second metatarsal heads and the big toe is about $64 \%$ of the total forefoot load. Based on published materials, Perttunen ${ }^{(1)}$ reviewed and summarized the values and the locations of the peak pp during walking (Figure 2). These data show clearly that during foot movement, the peak PP is transformed rapidly from the heel to the forefoot. The regions under the heel, medial and central forefoot and hallux exhibited the highest PP (Fig. 1). Eight anatomical regions of the foot were defined to be used for plantar pressure distribution analysis, namely: M01 at the heel, M02 at the medial midfoot, M03 at the lateral midfoot, M04 at medial forefoot, M05 at the central forefoot, M06 at the lateral forefoot, M07 at the hallux, and M08 at the toes ${ }^{(13)}$. 


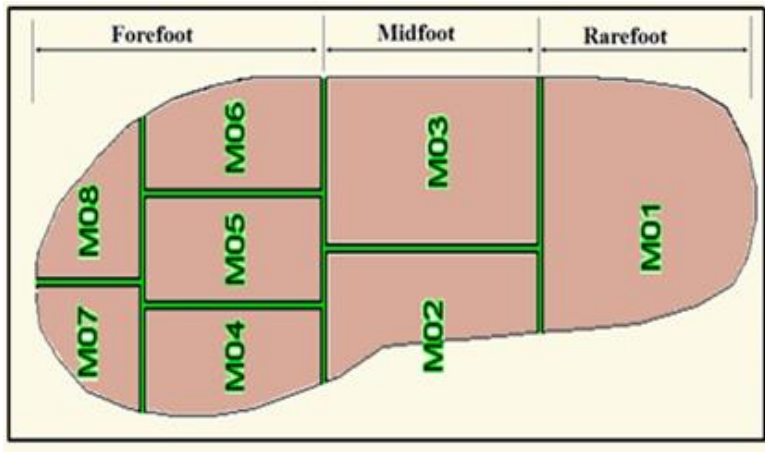

Fig. 1. Anatomical regions of the foot for plantar pressure distribution analysis ${ }^{(13)}$.

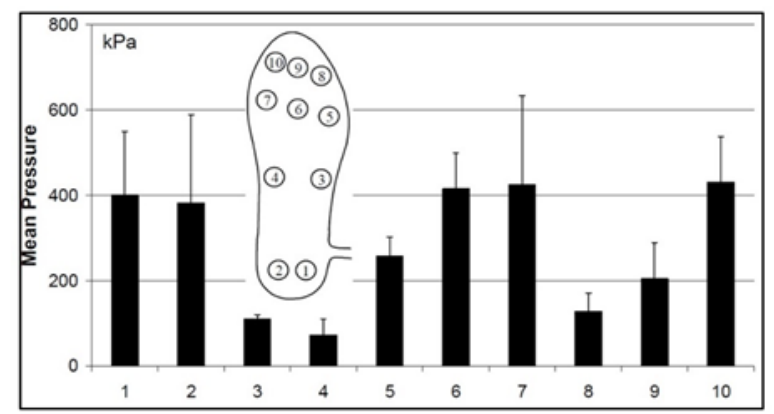

Fig 2. Mean and SD for the Peak Plantar Pressure during Walking $^{(1)}$.

As load distribution on the foot is affected by the foot shape, the foot can be described in three different categories by classifying the height of the foot arch. They are high foot arch (pes cavus), normal foot arch and low foot arch or flatfoot (pes planus) (Figure 3). Published literature has shown that the applied pressure underneath the plantar surface of flat-feet increases during foot movement, especially on the rearfoot, midial midfoot and lateral midfoot as compared with the normal foot ${ }^{(19)}$. Contact area and PP in midfoot noticeably decreases in individuals who have a high foot arch (cavus foot) ${ }^{(19)}$. Also, Chuckpaiwong ${ }^{(7)}$ studied the correlation between foot shape and the risk of plantar injuries and found that during foot movement, the stress and the peak PP concentrated on the fifth metatarsal of individuals with normal and high foot arch, whereas PP increases in the second and third metatarsal of the flat foot.

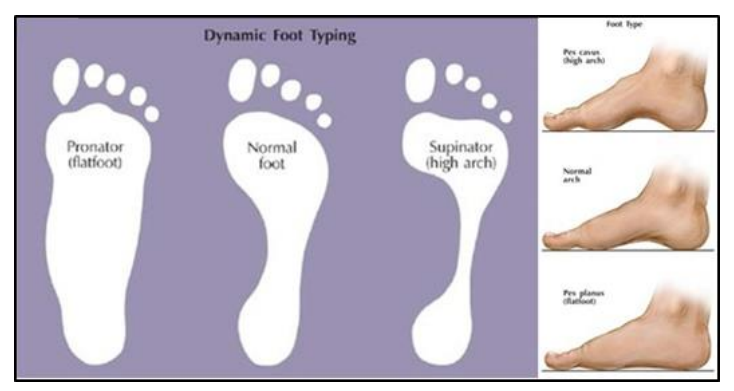

Fig. 3. Three different types of human foot arch and contact area of each type ${ }^{(35)}$.
As discussed in the above section, there are a number of factors that can alter the pressure/force distribution of the plantar surface. Therefore a good quality pressure sensing system is fundamental to the acquisition of high quality data for the evaluation and treatment of foot problems. In this investigation, a low-cost system was developed for static and dynamic precise plantar pressure measurement. This paper introduced the methods which include system calibration and testing using two adult subjects. The calibration and test results are provided to show the quality of the calibration and the measurement precision.

\section{Plantar Pressure Measuring Systems}

\subsection{Current Commercial Systems}

Generally, plantar load measuring systems can be classified into two main types: 1) PP measuring systems; and 2) ground reaction force (GRF) measuring system ${ }^{(20,21)}$. Currently, a number of measuring system products are commercially available. For example, foot insole and pressure $\operatorname{pad}^{(12,22,23)}$, Pedar ${ }^{(20,21)}$ and pressure platform systems $^{(20,25)}$ have been designed to record PP. In addition, force plate (e.g. AMTI) and wearable force sensors (e.g. the flexi-tactile sensor system Nitta Corporation) are commonly utilized to measure GRF of the moving foot ${ }^{(26,28)}$. These turnkey systems are expensive equipment.

Every foot-load measuring system used to measure plantar load or plantar pressure has some advantages and disadvantages ${ }^{(20,21)}$. Foot pressure measurement systems are electronic devices that are made to measure the pressure caused by interaction between the human foot and the support surface ${ }^{(8,21,29)}$. They can also be employed to determine the vertical forces $(\mathrm{Fz})$ experienced by human foot by integrating the individual pressure distributions ${ }^{(30)}$. Normally, foot pressure measurement systems consist of many small pressure sensors or pressure sensels. These sensors are ideal for measuring PP without disturbing subject or patient movement during trial because they are thin enough to enable non-intrusive measurement as shown in Figure $5^{(9,20,21,31)}$. These systems are useful devices in clinical gait analysis studies ${ }^{(21,25,31)}$, foot diagnoses and rehabilitation $^{(21)}$, footwear design, biomechanics analysis ${ }^{(25)}$ and other sport applications ${ }^{(32)}$. Therefore, a number of scientific studies have reported that pressure sensors can give sufficient results in analysing human gait compared to the more accurate data of the force plate ${ }^{(20)}$. However, the lack of accuracy (ranges approximately between $\pm 5 \%$ to $\pm 25 \%$ ), 
uncertainty and variations in the sensors readings, especially during the dynamic measurements, are the major limitations of pressure measurement systems ${ }^{(21)}$. In addition, one of the significant limitations associated with these systems is that they are reliant on force profile and do not relate the force distribution with movement of the foot ${ }^{(32)}$.

\subsection{Mat/pad System}

Mat/pad systems are constructed to be used for measuring static or dynamic pressure and a vertical force applied on a sole of the foot ${ }^{(21,32)}$. Generally, a platform system consists of many small electronic pressure sensors arranged in a matrix form on a circuit layer which is commonly inserted between two heat stable polyester layers (Figure 4). Mat resolution relies on the density of sensors in the pressure sensing mat (i.e. the resolution increase with increase the number of sensors). Using a mat/pad system for gait studies has a number of advantages. The system is ideal for measuring forces without disturbing the dynamics of test object as shown in Figure $4^{(21)}$. In addition, the high density (high resolution) of pressure sensors in a mat system allows for precise capturing and recording pressure information ${ }^{(32)}$.

Generally, a pressure mat system can record the vertical force as accurate as force plate recording because the mat system is usually placed on a flat surface parallel to the supporting ground surface similar to force platform system $^{(32)}$. Also, the sensor mat can be constructed in different resolutions and sizes with variety of shapes which can fit with specific clinical applications (e.g. assessing the pressure distribution between a patient's body and various supporting surfaces such as seats, backrests mattresses and cushions). Nevertheless, a number of studies indicate drawbacks associated with employing these systems for capturing foot pressure ${ }^{(30,32)}$. They generally require a large space with a proper setup because pressure mats need to be placed on a walkway to ensure the tested subject achieved a steady-state gait (minimum three steps are taken) before reaching the mat ${ }^{(30)}$. Also, to enhance the accuracy of the collected pressure data, the subject is required to repeat each trial (crossing the walkway) between 3 to 5 times which may be considered inappropriate and uncomfortable for patients who suffer from excessive ulcerations in their feet ${ }^{(32)}$.

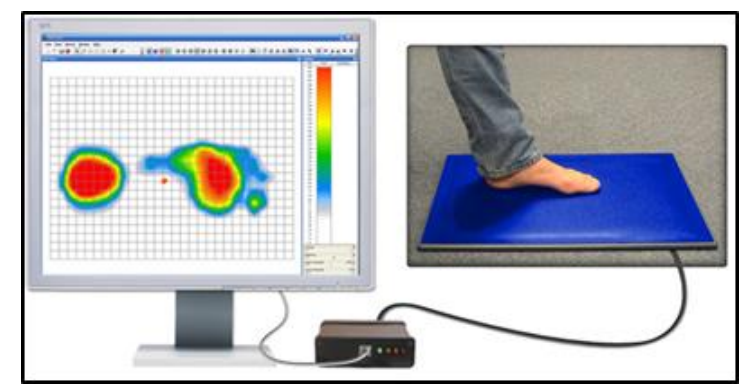

Fig. 4. A typical plantar pressure acquisition system. Note that the red color indicates high pressure zone.

\subsection{Sensor Calibration}

The electric resistance of a sensor is changed when a force is applied to $\mathrm{it}^{(20,25,29)}$. The loaded sensor gives readings either in ohms or in volt units ${ }^{(20,25)}$. Therefore, sensor calibration is an essential process to convert the sensor output data into pressure unit. It is also significant for determining the static and dynamic characteristics of the sensor (i.e. hysteresis, time drift, repeatability) which are necessary for applications that require continuous force measurement accuracy.

\subsubsection{Static Sensor Calibration Method}

This calibration technique is used to evaluate the response and the static properties of a sensor, e.g. hysteresis, creep, time drift, and repeatability ${ }^{(20,25)}$. Thus, it is suggested that each sensor of the foot pressure measurement systems is calibrated individually. Static calibration can be achieved either by inserting the sensors underneath a rubber bladder which is then filled with compressed air at various identified levels of pressure ${ }^{(32)}$ or by placing several known calibrated weights over the active area of pressure sensors ${ }^{(20)}$. The latter can be done either manually or automatically. The allowable range of the calibrated weights needs to be within the standard force sensitivity range of the sensors (e.g. between $0.1 \mathrm{~g}$ to $10 \mathrm{~N}$ ). Noce ${ }^{(20)}$, used an EnduraTEC test machine to apply nine different weights on each pressure sensor. These weights were placed in a random order for about one minute until they reached a steady reading of each sensor. After applying each weight the sensor was released for 1 minute to return to the normal condition.

Sensor response (output voltage) relative to the size of the applied load can be presented as load-resistance or loadconductance curves (Figure 5). The relationship between the applied load and the sensor output (voltage or ohms) should be a linear relationship ${ }^{(20,29)}$. A linearity shape in the loadconductance curve is desirable as this can make the calibration curve easy to use for calibration purposes. 
However, this relation typically is a non-linear curve (Figure $5 a)^{(20,21,25,29)}$ and several factors were recognized that might be responsible for producing the non-linearity of sensor outputs. These factors are: 1) the variations in sensitivity across the sensor; 2) the curvature of the contact surface; 3 ) temperature ; 4) humidity; 4) the materials of the insulating layers covering the sensor; and 5) creeping in the sensor outputs with constant applied load. With a non-linear curve, a simple linear regression approach is required to draw a best fitting line (Figure 5b).

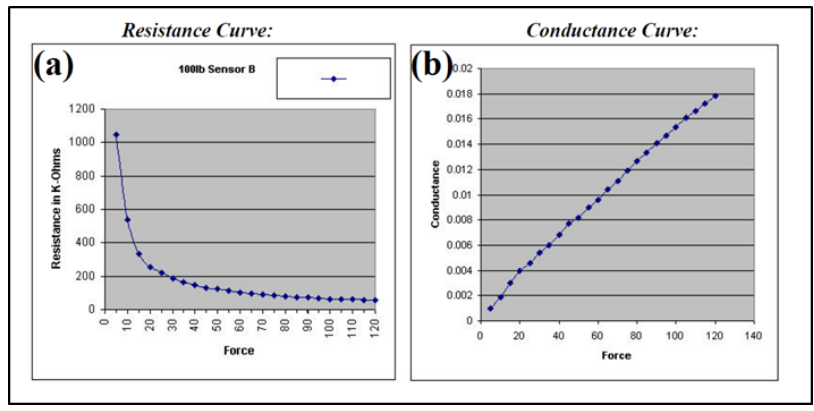

Fig. 5. (a) load-resistance; (b) load-conductance curves (Flexiforce Sensors User Manual).

\subsubsection{Dynamic Sensor Calibration Method}

Pressure measurement systems are also utilized for recording the pressure of moving objects such as gait and running. The dynamic performance of these systems requires evaluation in advance. Dynamic sensor calibration evaluates the sensitivity and accuracy of sensor responses to dynamic loads that are applied across the sensor ${ }^{(20,21,29)}$. The accuracy and the repeatability of the sensors' measurements can be analyzed during cyclic loading conditions. Dynamic calibration can be carried out by a number of techniques. Recently, a number of Authors ${ }^{(20,26,29)}$ developed an efficient and precise dynamic calibration method. The technique relies on utilizing an Instron materials testing machine (Figure 6) for generating an identified dynamic force varying over the time. The range of the produced forces should be within the force sensitivity range of each sensor. In this method, the Instron machine is programmed to load each individual sensor or mat of sensors with different cycles of loading based on the sensitivity range of each sensor as fast as possible ${ }^{(20,26)}$. However, Kärki ${ }^{(33)}$ used a Mini-Shaker (Figure 7) to provide dynamic excitation forces for sensor calibration. The sensitivity of the sensors was investigated by applying a set of dynamic excitation forces altering from $0.1 \mathrm{~N}$ to $10 \mathrm{~N}$ to the active areas of the sensors. Subsequently, a number of researchers adopted this calibration method ${ }^{(34)}$.

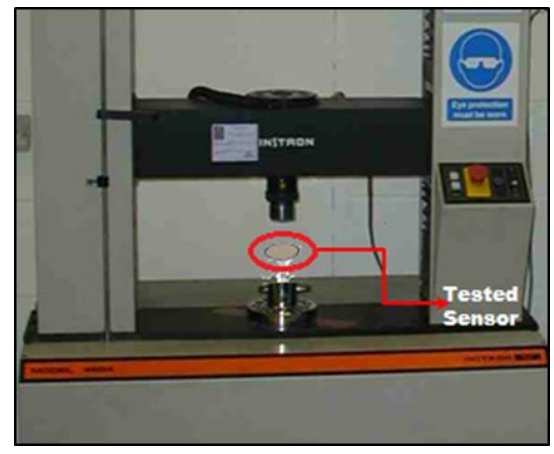

Fig. 6. Dynamic sensor calibration method using the Instron machine.

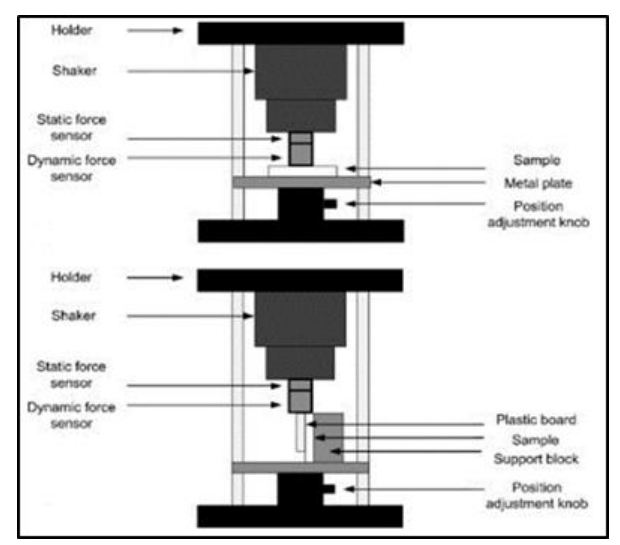

Fig. 7. Dynamic Sensor calibration method using MiniShaker ${ }^{(33)}$.

In a number of commercial plantar pressure measuring systems (e.g. Tekscan system) the sensor calibration consists of two processes: 1) sensor equilibration; and 2) sensor calibration. The first process requires minimizing or compensating the small variations (i.e. hysteresis, time drift, non-linearity, creep, and repeatability) between the sensing elements on any sensor. These are variations result from unique characteristics of individual sensors. In the second process, raw digital output data of the pressure sensor are converted to actual pressure units such as (Newton). Based on recent works ${ }^{(20,25,36)}$, this study considers both processes as a single activity and is referred to as a sensor calibration process.

\section{Methodology}

\subsection{Assembly of the Pressure Mat}

Thin-sheet sensor pads manufactured by ShuntMode MatrixArray® were purchased for the fabrication of a $150 \mathrm{~cm}$ 
by $400 \mathrm{~cm}$ gait mat. Each sheet consists of 70 an array of 70 force sensing cells, 7 rows and 10 columns, allows the user to simultaneously measure force of every cell at once. The active area was approximately $5 \mathrm{~cm}$ by $7.5 \mathrm{~cm}$ (Figure 8 ). Therefore, each sensor has a resolution of $7 \mathrm{~mm}$ by $7 \mathrm{~mm}$ square area. 16 sheets were assembled into a large gait mat (Figure 9). Software was written to record the conductance and output these data as pressure values. Before the final assembly of the gait mat, the sensor of individual sheets were calibrated as shown Figure 9. Each sheet in the assembly was identified so that the output can be converted to correct pressure value.

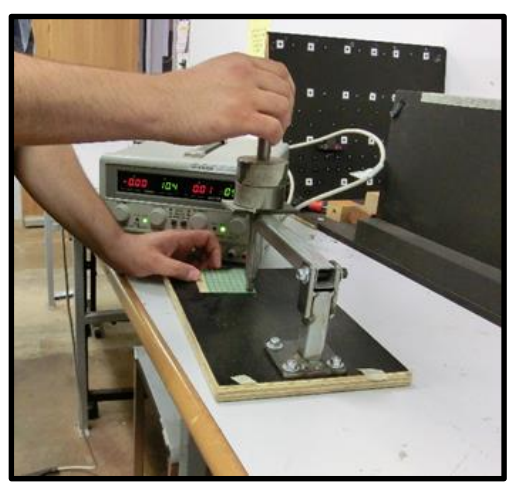

Fig. 8 Static calibration in the Gait Laboratory.

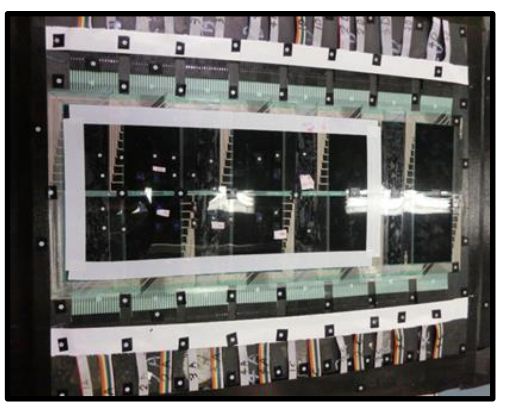

Fig. 9. The pressure mat consists of an array of small sensor pads.

\subsection{Human Gait Trial}

Two subjects (age: 35 and 58 years; height: 176 and 166 $\mathrm{cm}$; weight:90 and $63 \mathrm{~kg}$ ) with differing foot arch characteristics were recruited and volunteered for the study. The study was approved by the University Human Research Ethics Committee. The subjects' feet were marked with anthropometric lines and retro-reflective targets were attached to these markings as shown in Figure 10. After the preparation, the subjects were instructed to walk the normally over the gait mat (Figure 10). In the figure, (a) show the heel-strike while (b) shows the toe-off. The pressure between the plantar surface and the walking platform is outputted on the computer monitor screen and stored in the hard-drive.

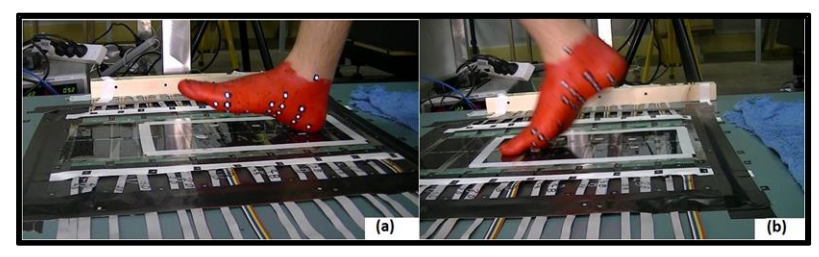

Fig. 10 Heel-strike (a) and toe-off (b) testing.

\section{Results and Analysis}

\subsection{Sensor Calibration}

Figure 11 shows the mathematical relationship between the conductance and the force applied to each sensor (sensor \#11 and sensor \#99). Two trend lines (linear and polynomial line) were plotted in the graph to show the conductance-force relationship. It is apparent that polynomial function should be used to produce the correct force value.
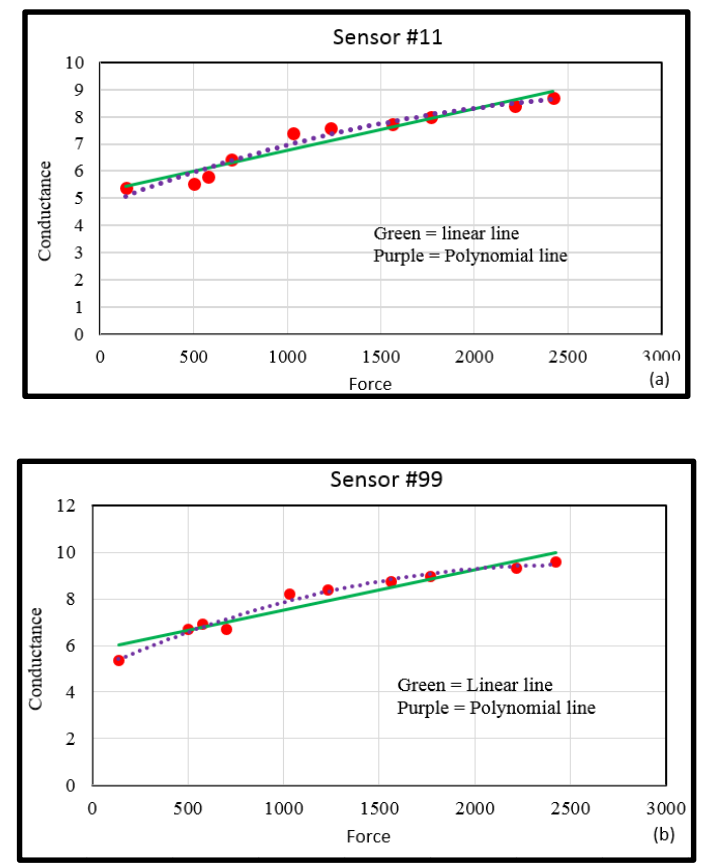

Fig. 11. Conductance curve for sensor \#11 and sensor \#99. Note the polynomial curves similarity in both graphs.

\subsection{Plantar pressure}

Figure 12 shows the captured plantar pressure 
characteristic in term of color contour. The figure depicts the sensor location, the pressure experienced at the individual sensor and a color chat showing the pressure at newton resolution. The dark blue contour indicates the lowest pressure zone while the bright red contour depicts the highest pressure zone. It is apparent that high pressure zone appeared at the mid-heel (heel-strike), the forefoot (midstance) and the large toe (toe-off). These features can be observed by a commercial plantar pressure acquisition system as shown in Figure 4. The shape of the foot-print indicates a high-arc foot. The capture pressure data was compared with a commercial system and the correlation of the vertical GRF $\left(\mathrm{F}_{\mathrm{z}}\right)$ captured by both systems was $r^{2}=0.92$. The averaged measurement and precision of the prototype is $5.5 \pm 1.8 \mathrm{~N} / \mathrm{cm}^{2}$ whereas a leading commercial system has a corresponding value of $2.5 \pm 1.0 \mathrm{~N} / \mathrm{cm}^{2}$. Nevertheless, the accuracy and resolution are still better than a number of off-the-self systems, thus the prototype is considered suitable for plantar pressure measurement.

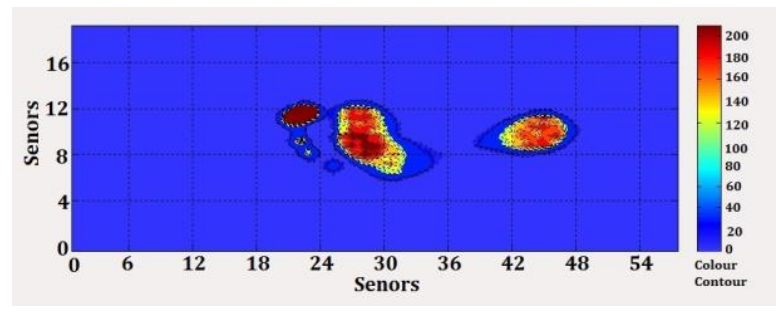

Fig. 12. Plantar pressure characteristic of Subject A shown in color contours.

\subsection{Prototype costing}

The cost of fabrication which includes materials and software writing was \$AUD $\$ 4,500$. A turn-key commercial system can be purchased for \$AUD15,000. However, many turn-key systems can be operated in wireless mode which is ideal for this type of application. The use of wireless of technology can increase the cost of the prototype by \$AUD1,500.

\section{Conclusions}

The research set out to develop a low-cost precision plantar pressure measuring system. The aim was to determine the suitability of such system for static and dynamic human gait and running applications. A literature review shows that such system is crucial for foot disease study and treatment, particularly determining the anomaly of individual gait characteristics.
In the research, a full-size gait pressure mat was fabricated in an Institutional Engineering Laboratory and software was written to convert sensor conductance into pressure data in Newton. The system was designed to show the acquired data displayed on the computer monitor screen in real-time and having the data stored in the computer for further processing.

The sensor calibration results show that polynomial mathematical function was required to convert the electrical output into force unit in Newton. Test results of two human subjects' slow gait trial show that the low-cost system developed provided precise plantar pressure data for the most demanding gait analysis applications.

The system developed is suitable for a range of health and industrial applications. Future research will involve evaluating the system developed for clinical work.

\section{Acknowledgment}

The authors thank the technical coordinator Mr Dean Beliveau and his technical staff at the Faculty of Engineering and Surveying, University of Southern Queensland, Australia for the works carried out in the assembly of this low-cost pressure mat system.

\section{References}

(1) Perttunen, J 2002, 'Foot loading in normal and pathological walking', $\mathrm{PhD}$ thesis, University of Jyväskylä, Finland.

(2) Castro, M, Abreu, S, Sousa, H, Machado, L, Santos, R \& Vilas-Boas, JP 2013, 'Ground reaction forces and plantar pressure distribution during occasional loaded gait', Applied Ergonomics, vol. 44, no. 3, pp. 503-9.

(3) Giacomozzi, C \& Martelli, F 2006, 'Peak pressure curve: an effective parameter for early detection of foot functional impairments in diabetic patients', Gait \& Posture, vol. 23, no. 4, pp. 464-70.

(4) Mao, D, Li, J \& Hong, Y 2006, 'The duration and plantar pressure distribution during one-leg stance in Tai Chi exercise', Clinical Biomechanics, vol. 21, no. 6, pp. 6405.

(5) Menz, HB \& Morris, ME 2006, 'Clinical determinants of plantar forces and pressures during walking in older people', Gait \& Posture, vol. 24, no. 2, pp. 229-36.

(6) Taylor, AJ, Menz, HB \& Keenan, A-M 2004, 'The influence of walking speed on plantar pressure measurements using the two-step gait initiation protocol', The Foot, vol. 14, no. 1, pp. 49-55. 
(7) Chuckpaiwong, B, Nunley, JA, Mall, NA \& Queen, RM 2008, 'The effect of foot type on in-shoe plantar pressure during walking and running', Gait \& Posture, vol. 28, no. 3, pp. 405-11.

(8) Martinez-Nova, A, Huerta, JP \& Sanchez-Rodriguez, R 2008, 'Cadence, age, and weight as determinants of forefoot plantar pressures using the Biofoot in-shoe system', Journal of the American Podiatric Medical Association, vol. 98, no. 4, pp. 302-10.

(9) Rodrigo, AS, Goonetilleke, RS \& Xiong, S 2013, 'Load distribution to minimise pressure-related pain on foot: a model', Ergonomics, vol. 56, no. 7, pp. 1180-93.

(10) Hayafune, N, Hayafune, Y \& Jacob, H 1999, 'Pressure and force distribution characteristics under the normal foot during the push-off phase in gait', The Foot, vol. 9, no. 2, pp. 88-92.

(11) Chang, H-W, Lin, C-J, Kuo, L-C, Tsai, M-J, Chieh, H-F $\&$ Su, F-C 2012, 'Three-dimensional measurement of foot arch in preschool children', Biomed Eng Online, vol. 11, p. 76.

(12) Vereecke, E, D'Août, K, De Clercq, D, Van Elsacker, L \& Aerts, P 2003, 'Dynamic plantar pressure distribution during terrestrial locomotion of bonobos (Pan paniscus)', American Journal of Physical Anthropology, vol. 120, no. 4, pp. 373-83.

(13) Ho, I-J, Hou, Y-Y, Yang, C-H, Wu, W-L, Chen, S-K \& Guo, L-Y 2010, 'Comparison of plantar pressure distribution between different speed and incline during treadmill jogging', Journal of Sports Science \& Medicine, vol. 9, no. 1, p. 154.

(14) Aydos, L, Uzun, A, Kaya, M, Kanatli, U, Esen, E \& Uslu, S 2012, 'The Effect of Volleyball on The Sole Contact Areas and Maximal Forces of Female Volleyball Players', Homo Sporticus, vol. 14, no.1, pp.5-11.

(15) Antunes, P, Dias, G, Coelho, A, Rebelo, F \& Pereira, T 2008, 'Non-linear finite element modelling of anatomically detailed 3D foot model', Report paper, pp. $1-11$.

(16) Cheng, H-YK, Lin, C-L, Wang, H-W \& Chou, S-W 2008, 'Finite element analysis of plantar fascia under stretch-the relative contribution of windlass mechanism and Achilles tendon force', Journal of Biomechanics, vol. 41, no. 9, pp. 1937-44.

(17) Hennig, EM, Staats, A \& Rosenbaum, D 1994, 'Plantar pressure distribution patterns of young school children in comparison to adults', Foot \& Ankle International, vol. 15, no. 1, pp. 35-40.

(18) Hayafune, N, Hayafune, Y \& Jacob, H 1999, 'Pressure and force distribution characteristics under the normal foot during the push-off phase in gait', The Foot, vol. 9, no. 2, pp. 88-92.

(19) Queen, RM, Mall, NA, Nunley, JA \& Chuckpaiwong, B 2009, 'Differences in plantar loading between flat and normal feet during different athletic tasks', Gait \& Posture, vol. 29, no. 4, pp. 582-6.

(20) Noce, HP 2005, 'An in-shoe instrument for acquisition and storage of plantar pressure', MSc thesis, Oregon State University, USA.

(21) Abdul Razak, AH, Zayegh, A, Begg, RK \& Wahab, Y 2012, 'Foot plantar pressure measurement system: A review', Sensors, vol. 12, no. 7, pp. 9884-912.

(22) Kalamdani, AA 2006, 'Development and characterization of a high-spatial-temporal-resolution foot-sole-pressure measurement system', MSc thesis, Carnegie Mellon University, Pittsburgh ,USA.

(23) de Souza, VA 2007, 'Design of Insole using Image Base Analysis', MSc thesis, The University of Tokyo, Japan.

(24) Muro-de-la-Herran, A, Garcia-Zapirain, B \& MendezZorrilla, A 2014, 'Gait Analysis Methods: An Overview of Wearable and Non-Wearable Systems, Highlighting Clinical Applications', Sensors, vol. 14, no. 2, pp. 336294.

(25)Rana, N 2009, 'Application of force sensing resistor (FSR) in design of pressure scanning system for plantar pressure measurement', Proceeding of the 2nd International Conference on Computer and Electrical Engineering 28-30 December, Dubai, UAE, pp. 678685.

(26) Liu, T, Inoue, Y \& Shibata, K 2009, 'A small and lowcost 3-D tactile sensor for a wearable force plate', Sensors Journal, IEEE, vol. 9, no. 9, pp. 1103-10.

(27) Rupérez, M, Monserrat, C \& Alcañíz, M 2009, 'A study of the viability of obtaining a generic animation of the foot while walking for the virtual testing of footwear using dorsal pressures', Journal of Biomechanics, vol. 42, no. 13, pp. 2040-6.

(28) Rupérez Moreno, MJ 2011, 'Multidisciplinary techniques for the simulation of the contact between the foot and the shoe upper in gait: virtual reality, computational biomechanics, and artificial neural networks', PhD thesis, Polytechnic University of Valencia, Spain

(29)Flórez, J \& Velasquez, A 2010, 'Calibration of force sensing resistors (fsr) for static and dynamic applications', IEEE ANDESCON Conference, 15-17 September 2010, Bogota, Colombia, pp.1-6. 
(30) Shi, BL 2001, 'Improving accuracy of the F-Scan sensor', Simulation, Engineering, and Testing Group, Jaycor, Inc., San Diego, CA. Report, pp. 3150-32.

(31) Bryant, J, Stevenson, J, Pelot, R, Morin, E, Deakin, J 2005. 'Development of a dynamic biomechanical model for load carriage: phase 4, part C3: dynamic assessment of pressure measurement systems for use in human load carriage', Queen's Univ Kingston (Ontario) Ergonomics Research Group, Toronto, Canada, pp.1-12.

(32) Orlin, MN \& McPoil, TG 2000, 'Plantar pressure assessment', Physical Therapy, vol. 80, no. 4, pp. 399409.

(33) Kärki, S, Lekkala, J, Kaistila, T, Laine, H-J, Mäenpää, H \& Kuokkanen, H 2009, 'Plantar pressure distribution measurements: An approach to different methods to compute a pressure map', Age (Years), vol. 23, no. 3, pp. 19-30.

(34) Paajanen, M, Lekkala, J \& Kirjavainen, K 2000, 'ElectroMechanical Film (EMFi)-a new multipurpose electret material', Sensors and Actuators A: Physical, vol. 84, no. 1, pp. 95-102.

(35) Kraushaar, D., 2005, 'Finding your running Shoe Matching foot type to shoe type', Hughston Research \& Education Journal of Athletic Training, <http://www.hughston.com/hha/a_17_2_4.htm>.

(36) Sumiya, T, Suzuki, Y, Kasahara, T \& Ogata, H 1998, 'Sensing stability and dynamic response of the F-Scan in-shoe sensing system: a technical note', Journal of Rehabilitation Research and Development, vol. 35, no. 2, pp. 192-200. 